\title{
Omalizumab in middle-aged or older patients with severe allergic asthma-COPD overlap
}

\author{
Luisa Ricciardi ${ }^{1}$, Francesco Papia ${ }^{1}$, Marta Liotta ${ }^{1}$, Francesca Cicero ${ }^{1}$, Stefania Isola ${ }^{1}$, Gennaro Tartarisco ${ }^{2}$, \\ Fabiana Furci ${ }^{1}$, Sebastiano Gangemi ${ }^{1}$
}

${ }^{1}$ Allergy and Clinical Immunology Unit, University of Messina, Messina, Italy

${ }^{2}$ National Research Council of Italy (CNR) - Institute for Biomedical Research and Innovation (IRIB), Messina, Italy

Adv Dermatol Allergol 2022; XXXIX (1): 88-93

DOI: https://doi.org/10.5114/ada.2022.113804

\begin{abstract}
Introduction: Biological therapies used for severe asthma may be useful even for middle-aged or older patients who have a history of severe allergic asthma with a chronic obstructive pulmonary disease (COPD) overlap phenotype. Aim: To show omalizumab efficacy in severe allergic asthma-COPD overlap disease.

Material and methods: We report our data of a retrospective study on 11 patients (mean age: 67.18 years) with a positive history of severe allergic asthma treated with omalizumab. They all presented limited reversibility of airway obstruction and signs of chronic bronchitis at radiological examinations, as in asthma-COPD overlap. Omalizumab improved conditions in terms of reduced exacerbations as well as asthma control test (ACT) and Asthma Quality of Life Questionnaire (AQLQ) scores.

Results: Clinical improvement was seen already in the first year with significantly increased ACT scores $(p<0.0001)$ and a significantly decreased number of exacerbations $(p<0.001)$. Furthermore, our data showed a significant inverse correlation over time between the number of exacerbations and ACT $(r=-0.83, p<0.0001)$, AQLQ symptoms $(r=-0.87, p<0.0001)$, forced expiratory volume in $1 \mathrm{~s}\left(\mathrm{FEV}_{1}\right)(r=-0.71, p<0.001)$ and $\mathrm{FEV}_{1} /$ forced vital capacity (FVC) $(r=-0.43, p=0.04)$. There also was a positive correlation between ACT and FEV $(r=0.74, p<0.0001)$, ACT and AQLQ symptoms $(r=0.93, p<0.0001)$, $\mathrm{FEV}_{1}$ and AQLQ symptoms $(r=0.67, p<0.001)$. All parameters continued to improve during the second year of treatment.

Conclusions: Omalizumab may be relevant as a therapeutic option even in middle-aged and older patients with severe asthma.
\end{abstract}

Key words: severe allergic asthma-COPD overlap, middle aged-older patients, omalizumab.

\section{Introduction}

The term "asthma-chronic obstructive pulmonary disease (COPD) overlap" has been suggested to be used in patients commonly seen in clinical practice with features of asthma and COPD [1].

Asthma and COPD include several different phenotypes, such as eosinophilic COPD, asthma with smoking and severe asthma in older patients with incomplete airflow reversibility or COPD patients who have a significant response to bronchodilators [2]. Patients with features of both asthma and COPD are treated as patients with asthma, with inhaled corticosteroids (ICS) in association to long-acting bronchodilators ( $L A B A$ ), to reduce the risk of severe exacerbations and death. Treatment with LABA in monotherapy or in association with long-acting mus- carinic agents (LAMA) even if indicated in COPD, could increase the risk of severe life-threatening exacerbations in asthma [3, 4].

Asthma and COPD overlap (ACO) in middle-aged or older patients may have a common underlying mechanism as T2 inflammation involving Th2, eosinophils and in general allergic pathways $[5,6]$. It has been reported that differential diagnosis of asthma, COPD and ACO could be exploited measuring fractional exhaled nitric oxide (FENO) as this is a biomarker of airway inflammation; therefore FENO is expected to be increased in asthma and ACO patients rather than in COPD patients [7].

Biological therapies which target these pathways are used for the treatment of severe refractory asthma but for asthma-COPD overlap data are needed [8].

Address for correspondence: Prof. Luisa Ricciardi MD, PhD, Allergy Unit, University of Messina, G. Martino Medical School Hospital, Via Consolare Valeria, 1. 98124 Messina, Italy, e-mail: Iricciardi@unime.it Received: 4.08.2020, accepted: 16.10.2020. 
Omalizumab is an effective therapy for patients with severe allergic asthma binding to serum IgE and reducing mast cell, basophil, dendritic and B cell responses [9-11]. Furthermore, it has been shown to reduce the bronchial reticular basement membrane thickness and eosinophil infiltration, typical of severe asthma re-modelling [12].

\section{Aim}

As to date there have been limited data available on the efficacy of omalizumab in populations with overlapping asthma and COPD, we report our experience with omalizumab in eleven patients with a positive history of allergic severe asthma due to sensitization to at least one perennial allergen, who started treatment with a limited reversibility of airway obstruction and signs of chronic bronchitis at radiological examination.

\section{Material and methods}

We present a retrospective study on 11 patients (6 females and 5 males), who were referred to our Allergy Unit for a positive history of allergic asthma with frequent exacerbations.

All eleven patients were non-smokers, had developed asthma in childhood, adolescence or young adulthood and were now either middle aged or older (mean age: 67.18 years). They had asthma symptoms with cough, hoarseness, intermittent chest tightness, daily and nocturnal wheezing, reported activity limitations because of dyspnoea and had needed emergency department visits for asthma exacerbations. Other chronic disorders, such as heart failure or lung diseases, as well as asthma comorbidities were not present. Asthma treatment consisted in high-dose ICS + LABA but patients reported $2 \geq$ exacerbations/year requiring OCS. Spirometry tests showed persistent airflow limitations as the forced expiratory volume in $1 \mathrm{~s}\left(\mathrm{FEV}_{1}\right)$ was $<80 \%$ of the predicted value and the bronchodilation test negative as the $\mathrm{FEV}_{1}$ did not increase by at least $12 \% / 200 \mathrm{ml}$ within $30 \mathrm{~min}$ after inhalation of $400 \mu \mathrm{g}$ of salbutamol.

All patients had positive allergic skin tests to perennial allergens of the Mediterranean area, either house dust mites or Parietaria judaica pollens; total IgE levels were $35-1500 \mathrm{KU} / \mathrm{l}$.

Therefore, as all 11 patients had a history of longstanding allergic asthma and presented persistent airflow limitations, the diagnosis was of asthma-COPD overlap with an allergic phenotype. Omalizumab treatment was started by calculating the necessary dose of omalizumab for each individual patient according to the baseline weight and total IgE levels. Omalizumab injections were given subcutaneously with a maximum dose of $600 \mathrm{mg}$ twice monthly.

Patients' medical history in the previous year, before starting omalizumab treatment, was collected, includ- ing disease status, current treatment, lung function and medical resource use.

Data were collected over a 2-year period: all patients were evaluated at T0, before starting omalizumab treatment, $\mathrm{T} 1$ (at 1 year) and T2 (at 2 years) in terms of Asthma Control Test (ACT), a 5 -item quick test that provides a numerical score to assess asthma control in the previous 4 weeks (a lower score represents uncontrolled asthma), number of exacerbations, $\mathrm{FEV}_{1}, \mathrm{FEV}_{1} / \mathrm{FVC}$, that is the ratio which represents the proportion of vital capacity that can be exhaled in the first second of forced expiration and Asthma Quality of Life Questionnaire (AQLQ) scores. The AQLQ score is the mean of 32 disease-specific quality of life questions which relate to the previous 2 weeks and assess four domains including symptoms, activity limitations, emotional function and environmental stimuli, with a lower AQLQ representing greater impairment of the health status.

All eleven patients, therefore, had the characteristics of patients with ACO.

Primary outcome was the change in symptom control measured by $A C T$, reduction in the number of exacerbations, increase in AQLQ scores and increase in FEV , FEV1/ FVC parameters.

The study was approved by the Ethics Committee of the University Hospital "G. Martino" of Messina, Italy (registration number 15/19).

\section{Statistical analysis}

Data analyses were carried out using SPSS 22 (IBM Corporation, Armonk, NY, USA) and R v3.6.1. Descriptive parameters are expressed as median and interquartile range (IQR). Differences in $A C T, F V_{1}, F E V_{1} / F V C$, number of exacerbations and AQLQ were analysed using the Friedman test and Wilcoxon test for paired non-parametric data. To evaluate the overall correlation, we used the repeated measures correlation [13-15]. Results were considered statistically significant with $p<0.05$.

\section{Results}

No significant differences and correlations in gender and age were found in our population of patients with ACO treated with omalizumab. Routine blood tests were within normal range, including blood count, liver and renal function and inflammation markers. All 11 patients presented statistically significant improvement of severe allergic asthma for values of ACT, number of exacerbations, FEV ${ }_{1}$ and AQLQ symptoms, limitations, emotive functions and environmental stimuli from T0 to T2. Only FEV1/FVC did not increase in a statistically significant manner. All differences of parameters between T0, T1 and T2 are reported in Table 1.

All 11 patients followed omalizumab treatment regularly with complete adherence to the prescribed schedule.

The ACT score increased significantly from TO to T1 $(p<0.0001)$, T1 to T2 $(p<0.01)$ and the number of exac- 
Table 1. Median values and interquartile ranges (IQR) extracted for T0, T1, T2 for ACT, AQLQ, exacerbations, FEV , $\mathrm{FEV}_{1} / \mathrm{FVC}$ parameters and $p$-value of non-parametric Friedman test

\begin{tabular}{lccccc}
\hline Parameter & T0 & T1 & T2 & Test & $P$-value \\
\hline ACT & $11(2.5)$ & $18(3)$ & $20(1.5)$ & Friedman & $<0.0001$ \\
\hline No. of exacerbations & $25(23)$ & $3(5.5)$ & $1(3)$ & Friedman & $<0.0001$ \\
\hline FEV ${ }_{1}$ FEV & $55(15.5)$ & $59(17.5)$ & $65(21)$ & Friedman & $<0.01$ \\
\hline FEL $/$ FVC & $84(15.5)$ & $86(21)$ & $87(24.5)$ & Friedman & 0.16 \\
\hline AQLQ activity limitations & $21(4.5)$ & $46(12)$ & $53(9.5)$ & Friedman & $<0.0001$ \\
\hline AQLQ emotive functions & $26(4)$ & $45(9.5)$ & $48(9)$ & Friedman & $<0.0001$ \\
\hline AQLQ environmental stimuli & $11(4)$ & $18(2)$ & $18(3)$ & Friedman & $<0.001$ \\
\hline
\end{tabular}

A

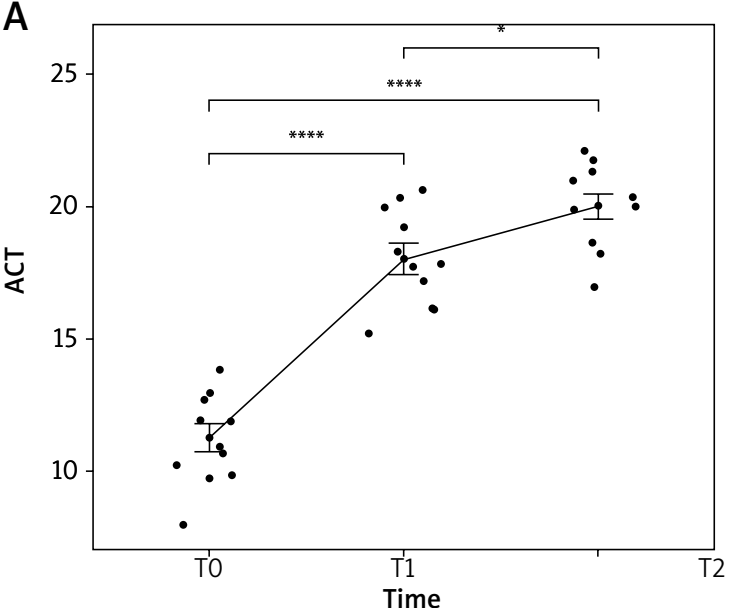

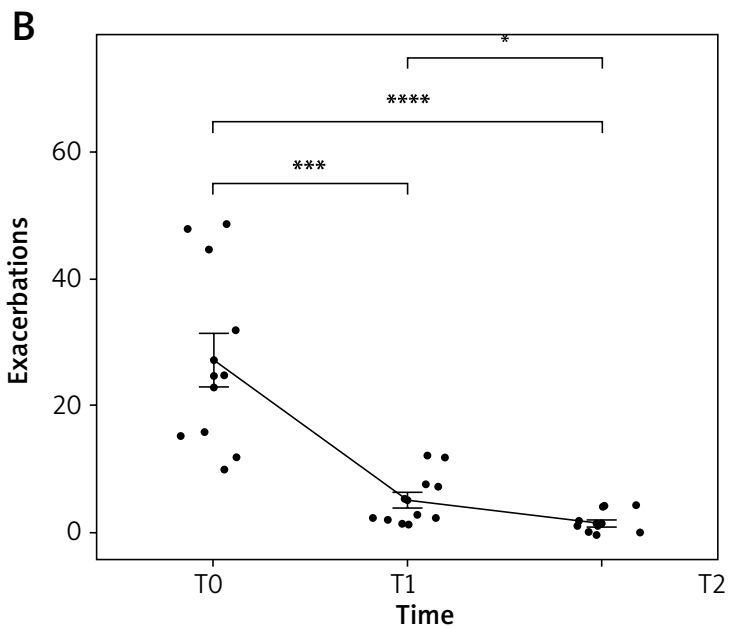

${ }_{* * *, * * *}$ and ${ }^{* * * *}$ indicate significance of Wilcoxon rank sum test with p-value $<0.1,<0.01,<0.001$ and $<0.0001$.

Figure 1. Jitter plots shows ACT (A) and the number of exacerbations (B) measured at T0, T1 and T2
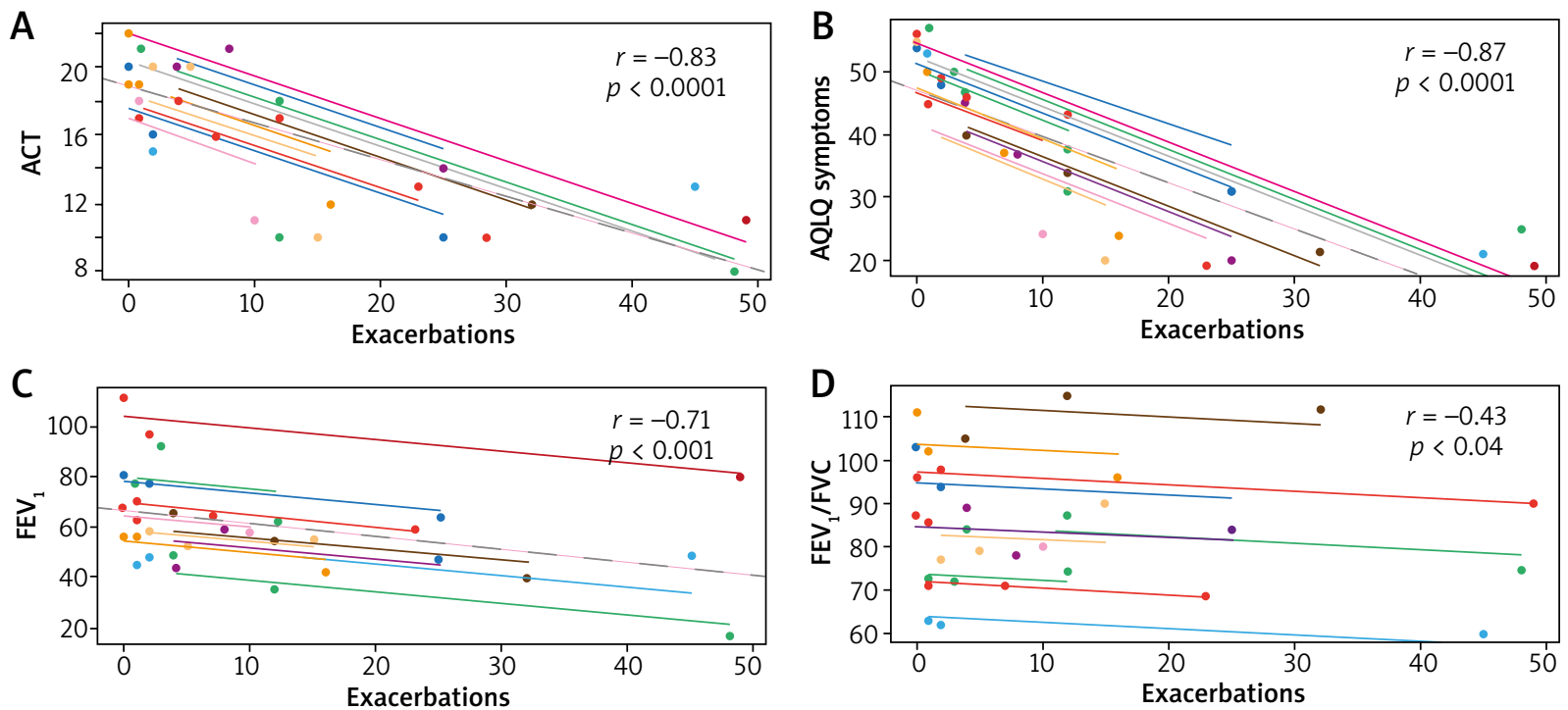

Figure 2. A - Overall correlation between exacerbations and ACT with $r=-0.83, p<0.0001$. B - Correlation between exacerbations and AQLQ symptoms with $r=-0.87, p<0.0001$. C - Overall correlation between exacerbations and FEV with $r=-0.71, p<0.0001$. D - Overall correlation between exacerbations and FEV, FVC with $r=-0.43, p=0.04$. Observations from the same participant are given in the same colour, with corresponding lines to show the linear fit of multiple measures (T0, T1, T2) for each participant 
A

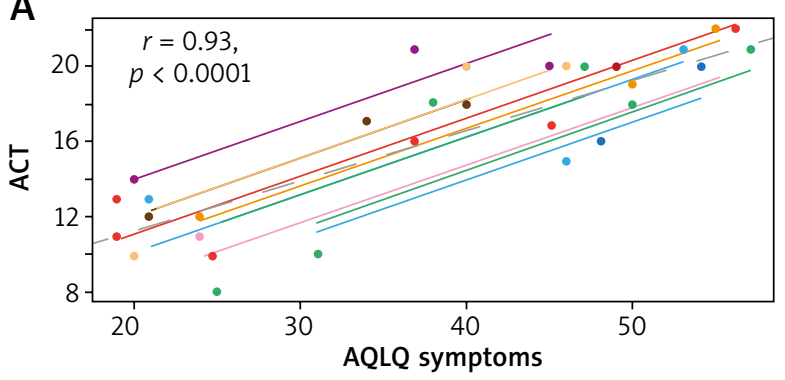

C

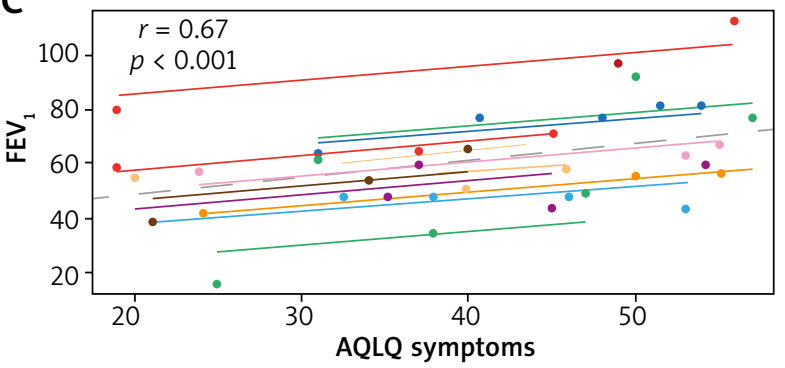

B

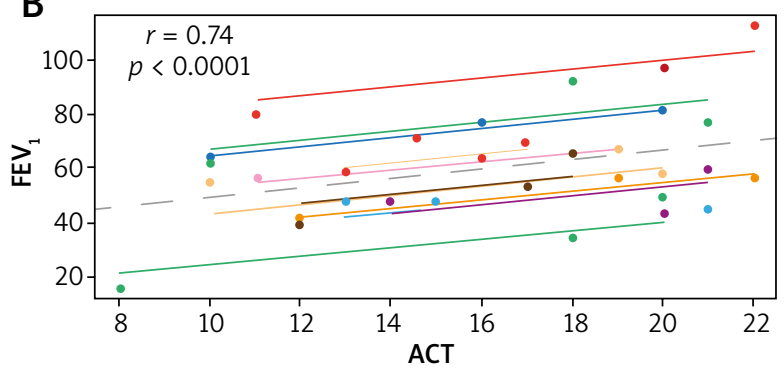

D

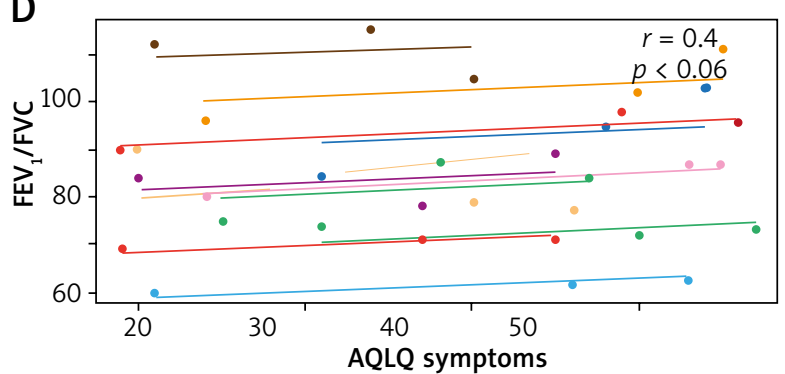

Figure 3. A - Overall correlation between AQLQ symptoms and ACT with $r=0.93, p<0.0001$. B - Correlation between ACT and FEV 1 with $r=0.74, p<0.0001$. C - Overall correlation between AQLQ symptoms and $\mathrm{FEV}_{1}$ with $r=0.67, p<0.001$. D - Overall correlation between AQLQ symptoms and FEV $/ F V C$ with $r=0.40, p=0.06$. Observations from the same participant are given in the same colour, with corresponding lines to show the linear fit of multiple measures (T0, T1, T2) for each participant

erbations decreased significantly over time from T0 to T1 $(p<0.001)$ and T1 to T2 $(p<0.01)$ as shown in Figure 1. In addition, all AQLQ parameters increased significantly from T0 to T1 ( $p<0.0001)$. No significant differences in $\mathrm{FEV}_{1}$ and $\mathrm{FEV}_{1} / \mathrm{FVC}$ were found over time. Additionally, a significant inverse correlation was observed over time between the number of exacerbations and ACT $(r=-0.83, p<0.0001)$, exacerbations and AQLQ symptoms $(r=-0.87, p<0.0001)$, exacerbations and FEV $(r=-0.71, p<0.001)$, exacerbations and $\mathrm{FEV}_{1} / \mathrm{FVC}(r=$ $-0.43, p=0.04)$ as shown in Figure 2. We also observed a positive correlation between AQLQ symptoms and ACT $(r=0.93, p<0.0001)$, ACT and $\mathrm{FEV}_{1}(r=0.74, p<0.000)$, AQLQ symptoms and $\mathrm{FEV}_{1}(r=0.67, p<0.001)$, AQLQ symptoms and $\mathrm{FEV}_{1} / \mathrm{FVC}(r=40, p=0.06)$ as shown in Figure 3.

Radiologic diagnostic tests, such as either chest $\mathrm{X}$-rays or computed tomography (CT) were performed at TO. Scan images showed signs of chronic bronchitis such as increased air trapping, typical of emphysema, or increased wall thickness, typical of fibrosis.

None of the 11 patients discontinued omalizumab during the 2-year follow-up due to adverse events. Overall omalizumab treatment was well tolerated with only slight pain in the injection site during subcutaneous injection in 2 female patients out of the 11 patients studied.

\section{Discussion}

Asthma-COPD overlap in middle-aged or older patients is characterized by airway obstruction typical of asthma and limited reversibility typical of COPD, therefore it is described as difficult to manage and phenotype [16].

At present it is treated with ICS/LABA in association with ipratropium bromide as a LAMA in add-on treatment but, according to severity, different therapeutic approaches are needed [17]. One specific treatment in the presence of allergic sensitization to perennial allergens, such as house dust mites [18] and in the Mediterranean area, Parietaria judaica pollens [19], is anti-lgE treatment with omalizumab [20].

Omalizumab is a recombinant humanized monoclonal anti-lgE antibody, which prevents free IgE from binding to their high and low affinity receptors on effector cells interfering with the allergic cascade in patients with severe allergic asthma [21].

While the role of IgE in severe asthma has already been well established, the role of omalizumab in COPD populations is still under study [21, 22]. Stoll et al. [23] showed how COPD and allergic asthma are characterized by a similar over-expression of the high-affinity IgE receptor on plasmacytoid dendritic cells. In another study [24], IL-17, a key role cytokine in asthma pathogenesis was higher in COPD patients. Moreover, Th2 inflammation 
might be present in COPD and, therefore, omalizumab treatment is beneficial in this population [25].

At present, data on omalizumab treatment in asthma-COPD overlap populations are limited as patients with the diagnosis of COPD are usually excluded from clinical trials $[26,27]$.

Furthermore, it is still difficult to define an asthmaCOPD overlap status in real-life settings [28]. The clinical diagnosis up to now was based on the positive history for asthma and lung function measurements with $\mathrm{FEV}_{1}$ / FVC $<0.7$ or $\mathrm{FEV}_{1}<80 \%$ of predicted value, in the presence of an obstructive spirometry airflow curve with a negative bronchodilation test [29]. The presence of a positive history for allergic respiratory diseases with sensitization to inhalant allergens can also help to distinguish asthma-COPD overlap from COPD [30].

In our study, omalizumab treatment was efficacious for patients with asthma-COPD overlap, non-responsive to ICS/LABA with LAMA as add-on treatments. The study data are on 11 patients, non-smokers, with the history of severe allergic asthma, positive specific IgE to perennial allergens such as dust mites and Parietaria judaica pollens with signs of COPD at chest X-rays or CT.

Our data showed that patients treated with omalizumab might improve their condition in terms of reduced

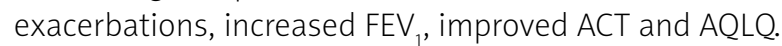
Clinical improvement, in our patients, correlated, especially after the first year of therapy, with significantly increased ACT scores $(p<0.0001)$ and significantly decreased number of exacerbations $(p<0.001)$. Furthermore, patients' quality of life significantly improved in the first year of omalizumab treatment as AQLQ scores significantly increased $(p<0.001)$. Spirometry parame-

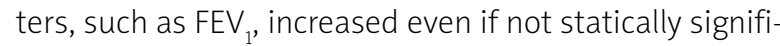
cantly, and no bronchial reversibility was achieved. Bronchodilator-mediated reversibility is usually proposed as a diagnostic criterion to differentiate asthma from COPD, but it does not rule out asthma-COPD overlap and does not consider that reversibility may not be present in severe asthma [5]. Nevertheless, in our study all real-life indicators, such as the number of exacerbations, ACT and AQLQ scores, significantly improved with omalizumab as an add-on therapy continuing to improve during the second year of treatment. Omalizumab, therefore, ensured that the goals of asthma-COPD overlap treatment were reached, such as prevention of exacerbations, symptoms relief and quality of life improvement.

Data reported in our study, unfortunately, have some bias as they were collected retrospectively and on a small group of patients. Nevertheless, they show the coexistence of asthma-COPD overlap in middle-aged or older patients refractory to conventional pharmacological therapies, sensitized to at least one perennial allergen and responders to omalizumab as an add-on treatment.

\section{Conclusions}

In our study, treatment with omalizumab resulted in asthma control and improved quality of life in a small population of middle-aged or older patients with asthmaCOPD overlap.

Future studies on omalizumab efficacy in populations with the same characteristics will provide additional insights for treatment of patients who have features of both asthma and COPD with an allergic phenotype.

\section{Conflict of interest}

The authors declare no conflict of interest.

\section{References}

1. Global Initiative for Asthma. Global Strategy for Asthma Management and Prevention, 2020. Available from www. ginasthma.org

2. Toledo-Pons N, van Boven JFM, Roman-Rodriquez M, et al. ACO: Time to move from the description of different phenotypes to the treatable traits. PLoS One 2019; 14: e0210915.

3. Grainge CL, Maltby S, Gibson PG, et al.Targeted therapeutics for severe refractory asthma: monoclonal antibodies. Expert Rev Clin Pharmacol 2016; 9: 927-41.

4. Jamieson DB, Matsui EC, Belli A, et al. Effects of allergic phenotype on respiratory symptoms and exacerbations in patients with chronic obstructive pulmonary disease. Am J Respir Crit Care Med 2013; 188: 187-92.

5. Parulekar AD, Diamant Z, Hanania NA. Role of biologics targeting type 2 airway inflammation in asthma: what have we learned so far? Curr Opin Pulm Med 2017; 23: 3-11.

6. Neves MC, Neves YC, Mendes CM, et al. Evaluation of atopy in patients with COPD. J Bras Pneumol 2013; 39: 296-305.

7. Takayama Y, Ohnishi H, Ogasawara F, et al. Clinical utility of fractional exhaled nitric oxide and blood eosinophils counts in the diagnosis of asthma-COPD overlap. Int I Chron Obstruct Pulmon Dis 2018; 13: 2525-32.

8. Barnes PJ. Therapeutic approaches to asthma-chronic obstructive pulmonary disease overlap syndromes. J Allergy Clin Immunol 2015; 136: 531-45.

9. Busse W, Corren J, Lanier BQ, et al. Omalizumab, anti-IgE recombinant humanized monoclonal antibody, for the treatment of severe allergic asthma. J Allergy Clin Immunol 2001; 108: 184-90.

10. Hanania NA, Alpan O, Hamilos DL, et al. Omalizumab in severe allergic asthma inadequately controlled with standard therapy: a randomized trial. Ann Intern Med 2011; 154: 57382.

11. Abraham I, Alhossan A, Lee CS, et al. "Real-life" effectiveness studies of omalizumab in adult patients with severe allergic asthma: systematic review. Allergy 2016; 71: 593-610.

12. Riccio AM, Dal Negro RW, Micheletto C, et al. Omalizumab modulates bronchial reticular basement membrane thickness and eosinophil infiltration in severe persistent allergic asthma patients. Int J Immunophatol Pharmacol 2012; 25: 475-84.

13. Bland JM, Altman DG. Calculating correlation coefficients with repeated observations: Part 2 - correlation between subjects. BMJ 1995; 310: 633. 
14. Bland JM, Altman DG. Statistics notes: calculating correlation coefficients with repeated observations: Part 1 - correlation within subjects. BMJ 1995; 310: 446.

15. Bakdash JZ, Marusich LR. Repeated measures correlation. Front Psycol 2017; 8: 456

16. Maselli DJ, Hanania NA. Management of asthma COPD overlap. Ann Allergy Asthma Immunol 2019; 123: 335-44.

17. Kondo M, Tamaoki J. Therapeutic approaches of asthma and COPD overlap. Allergol Int 2018; 67: 187-90.

18. Moore WC, Bleecker ER, Curran-Everett D, et al. Characterization of the severe asthma phenotype by the National Heart, Lung, and Blood Institute's Severe Asthma Research Program. J Allergy Clin Immunol 2007; 119: 405-13.

19. Sala-Cunill A, Bartra J, Dalmau G, et al. Prevalence of asthma and severity of allergic rhinitis comparing 2 perennial allergens: house dust mites and parietaria judaica pollen. I Invest Allergol Clin Immunol 2013; 23: 145-51.

20. Incorvaia C, Ridolo E. In the strategies to prevent asthma exacerbations, allergic asthma needs specific treatment. Curr Med Res Opin 2015; 31: 821-3.

21. Katial RK, Bensch GW, Busse WW, et al. Changing paradigms in the treatment of severe asthma: the role of biologic therapies. J Allergy Clin Immunol Pract 2017; 5: 1-14 https: doi: 10.1016/j.jaip.2016.11.029.

22. Sposato B, Scalese M, Milanese M, et al. Should omalizumab be used in severe asthma/COPD overlap? J Biol Regul Homeost Agents 2018; 32: 755-61.

23. Stoll P, Bähker A, Ulrich $M$, et al. The dendritic cell high-affinity IgE receptor is overexpressed in both asthma and severe COPD. Clin Exp Allergy 2016; 46: 575-83.

24. Yalcin AD, Celik B, Yalcin AN. Omalizumab (anti-lgE) therapy in the asthma-COPD overlap syndrome (ACOS) and its effects on circulating cytokine levels. Immunopharmacol Immunotoxicol 2016; 38: 253-6.

25. Tat TS, Cilli A. Omalizumab treatment in asthma-COPD overlap syndrome. J Asthma 2016; 53: 1048-50.

26. Caminati M, Senna G, Guerriero M, et al. Omalizumab for severe allergic asthma in clinical trials and real-life studies: what we know and what we should address. Pulm Pharmacol Ther 2015; 31: 28-35.

27. Maltby S, Gibson PG, Powell H, McDonald VM. Omalizumab treatment response in a population with severe allergic asthma and overlapping COPD. Chest 2017; 151: 78-89.

28. Yousuf A, Ibrahim W, Greening NJ, Brightling CE. Biologics for chronic obstructive pulmonary disease. J Allergy Clin Immunol Pract 2019; 7: 1405-16.

29. Matthes S, Stadler J, Barton J, et al. Asthma features in severe COPD: identifying treatable traits. Respir Med 2018; 145: 89-94.

30. Padrão E, Araújo D, Todo Bom A, et al. Asthma-COPD overlap: a Portuguese survey. Pulmonology 2018; 24: 174-81. 